\title{
A note on the Full Counting Statistics of paired fermions
}

\author{
Israel Klich \\ Department of Physics, University of Virginia, Charlottesville, VA, USA
}

\begin{abstract}
We study trace formulas for the exponentials of general fermion bilinears, including pairing terms, and including non Hermitian forms. In particular, we give elementary derivations for determinant and Pfaffian formulae for such traces, and use these to obtain general expressions for the full counting statistics in states associated with quadratic Hamiltonians, including BCS-like pairing terms and fermion parity in a prescribed region or set of modes. We also derive Pfaffian expressions for state overlaps and counting statistics in states built out of the vacuum by creation of pairs of particles.
\end{abstract}

\section{INTRODUCTION}

Fluctuations of observables in quantum mechanical systems carry important information about a variety of properties, such as the nature of transport mechanisms in mesoscopic systems or spin excitations in magnetic systems, and supply a tool for studying physical properties both in equilibrium and non-equilibrium settings. An important example for such is the use of shot noise, i.e. non-equilibrium current fluctuations, to get information about the nature of charge carriers in complicated interacting situations such as fractional quantum Hall systems, where collective excitations carry a fractional charge. Additional, more detailed information about condensed matter systems, may be hidden in higher order noise correlations. This information becomes accessible as our ability to carry out precision measurements increases. The full distribution functions of transmitted charges, known as the "Full Counting Statistics" (FCS) has been used to describe transport in mesoscopic systems, and involve interesting problems both about the nature of transport and the nature of measurements themselves.

In the context of mesoscopic physics, the theory of FCS was pioneered in the celebrated work of Levitov and Lesovik [1], where a beautiful formula for the FCS of transport through a junction has been derived. Subsequently, FCS has seen intense work in mesoscopic physics [1] 7 and in cold atom systems [8, 9]. The formulation of the FCS results for free fermions 
presented in [10], has been successfully used to simplify FCS and related calculations and has proved useful also in other contexts, describing time dependent problems involving noninteracting fermions. For example, the FCS formalism has been found to play a useful role in the Fermi-edge problem [11, 12], and has been useful as a numerical tool for other time dependent problems, for example in studies of thermalization and decoherence of metallic leads [13], as a tool to characterize correlations and phases [14], identify dynamical phases in the resonant-level model [15] as well as characterizing non-equilibrium situations such as the evolution of systems following a quench [16]. FCS was studied theoretically and measured experimentally in several mesoscopic systems, such as tunnel junctions and quantum point contacts [17 19] where non-gaussian fluctuations [18] and counting statistics of single electrons [20] were measured. Some attention has also been devoted to states where pairing terms appear in the Hamiltonian, to treat situations involving superconductivity. In such situations transitions between electron holes pairs and Cooper pairs via Andreev tunneling are present (see, e.g. [21 24]). Recent experimental progress has been reported in the measurements of FCS of Andreev tunneling in [25]. Shot noise signatures for systems with fractionalized charges have been proposed theoretically [26 28] and are used to experimentally access fractional charges [29 31]. On the mathematical side, much work has been done to understand the thermodynamic limit of FCS on a more rigorous level, see e.g [32 36$]$.

In a broader context, it has also been demonstrated that fluctuations, and the FCS of charge or other conserved quantities (such as, for example, block magnetization in certain spin chains), may contain information about the full entanglement scaling of a system split into two parts. It was shown how one can compute entanglement entropy and Renyi entropies from FCS for certain systems in [37], a measurement of entanglement entropy in a transport experiment using FCS was suggested in [38] and other systems were discussed in [39, 40].

Many of the above advances hinge on the efficient calculation of partition function like objects - traces of exponents of quadratic fermion Hamiltonians. However, paired Hamiltonians possess an additional layer of complexity compared to the non-interacting fermions, due to the lack of charge conservation. Algebraically, the lifting of single particle dynamics is non-unique, as the group of Bogolubov transformations is not simply connected, resulting in sign ambiguities.

Here we present a rather simple closed formula, Eq. (44), for the trace of a single exponent of quadratic fermion operators, where the sign ambiguity is not present. For products of 
exponents and most practical purposes, in the equations presented below, such as Eq. (27), the sign can be determined by simple analyticity arguments. In addition we supply simple formulas for full counting statistics and overlaps in pairing states as described in Eq. (62) and Eq. (63).

\section{THE TRACE OF THE EXPONENTIAL OF A FERMION BILINEAR}

In this section we show how to extend the well known formula for the partition function of a number conserving, bilinear, fermion Hamiltonian

$$
\operatorname{Tr} e^{-\beta H_{i j} a_{i}^{\dagger} a_{j}}=\operatorname{det}\left(1+e^{-\beta H}\right),
$$

to situations where the quadratic form includes pairing terms. As we will observe shortly, in working with Hamiltonians which include pairing terms it is convenient to represent the fermions using Majorana operators. To do so, consider an $n$-dimensional Hilbert space $\mathfrak{h}$ of possible single particle states (modes), and associated fermionic creation and annihilation operators $a_{k}^{\dagger}, a_{k}$, with $k=1, . ., n$, obeying the canonical anti-commutation relations: $a_{k}^{\dagger} a_{l}+$ $a_{l} a_{k}^{\dagger}=\delta_{l k}$ and $a_{k} a_{l}+a_{l} a_{k}=0$. We define $2 n$ Majorana operators by

$$
\begin{gathered}
c_{k}=a_{k}^{\dagger}+a_{k} ; \quad k=1, . ., n, \\
c_{k}=i\left(a_{k}^{\dagger}-a_{k}\right) ; k=n+1, . ., 2 n,
\end{gathered}
$$

with the $c_{k}$ obeying the Clifford relations:

$$
c_{k} c_{l}+c_{l} c_{k}=2 \delta_{k l} .
$$

To define taking traces below, we assume a standard representation of the Clifford algebra on the $2^{n}$ dimensional Fock space associated with the fermions. In practice we use the algebraic relations, and fix the dimension of the representation to $2^{n}$ (alternatively, one may represent the algebra on a $2^{n}$ dimensional Hilbert space explicitly in terms of Pauli matrices, augmented by Jordan-Wigner strings).

In this section we derive the following results:

1) For a $2 n$ dimensional, antisymmetric matrix $A \in \operatorname{Skew}(2 n, \mathbb{C})$, the following formula holds:

$$
\mathcal{Z}(A) \equiv \operatorname{Tr} e^{A_{i j} c_{i} c_{j}}=\frac{\operatorname{Pf}\left(e^{-4 A}-e^{4 A}\right)}{\operatorname{Pf}\left(e^{-2 A}-e^{2 A}\right)} .
$$


were Pf is the Pfaffian, defined for a $2 n \times 2 n$ matrix $M$ by

$$
\operatorname{Pf}(M)=\frac{1}{2^{n} n !} \Sigma_{\mathcal{P} \in \mathcal{S}_{2 n}}(-1)^{\mathcal{P}} M_{P_{1} P_{2}} M_{P_{2} P_{3}} \ldots M_{P_{2 n-1} P_{2 n}}
$$

here $\mathcal{S}_{2 n}$ is the permutation group on $2 n$ elements.

2) In addition, we note a simpler formula, which is correct up to a sign ambiguity:

$$
\mathcal{Z}(A)=\sqrt{\operatorname{det}\left(1+e^{4 A}\right)}
$$

3) Also, we note that the canonical mode decomposition available for Hermitian quadratic Hamiltonians, Eq. (12), may be extended to an arbitrary non-degenerate (possibly complex) antisymmetrix form $A_{i j} c_{i} c_{j}$ using a similarity transformation on Fock space, and into a Gantmacher mode decomposition, Eq. (19), when the degeneracy cannot be simply resolved.

Note the appearance of the square root in the determinant equation (6) . In this equation, the sign of the determinant has to be determined. In many practical calculations the sign can be determined as follows. Consider $\mathcal{Z}(\lambda A)$ : from it's definition, Eq. (4), it follows that $\mathcal{Z}(\lambda A)$ is an analytic function of $\lambda$. This determines the correct way of taking the sign of the square root: the sign has to be taken so that the right hand side of Eq. (6) is everywhere analytic as well, and so that at $\lambda=0$ we have $\mathcal{Z}(0)=2^{n}$, the dimension of the corresponding

Fock space. That also implies that any zeroes of $\operatorname{det}\left(1+e^{4 A}\right)$ must come in pairs, as to not create branch cuts in the complex $\lambda$ plane. In the next section we will repeat this type of argument with several other expressions involving square roots.

In particular, when $A$ is real antisymmetric, the equations (44) and (6) are expressions for the partition function of paired fermions with the (hermitian) Hamiltonian $A_{i j} c_{i} c_{j}$, in such a case the square root in Eq. (6) must be taken as positive.

\section{NORMAL FORMS IN FOCK SPACE AND DERIVATION OF FORMULAS}

To prove the above results, we need the following preliminary considerations. Consider $O \in O(2 n, \mathbb{C})$, a complex orthogonal transformation (i.e. $O^{T} O=1$ ). We emphasize that the group of complex orthogonal matrices is very different from the group of unitary matrices and the group of orthogonal matrices, in particular $O(2 n, \mathbb{C})$ is not compact. We note that for $O \in O(2 n, \mathbb{C})$ the transformation:

$$
c_{k} \rightarrow O_{k l} c_{l}
$$


preserves the anti-commutation relations (3)).

Next, we establish the important fact that this transformation can always be written as a similarity transformation on the Clifford algebra obeyed by the $c_{k}$, i.e. there is some $X(O)$, and operator acting on Fock space, such that $X(O) c_{k} X(O)^{-1}=O_{k l} c_{l}$. To see this, note that any complex orthogonal transformation can be written as:

$$
O=O_{R} e^{i K}
$$

where $O_{R}$ is a real orthogonal matrix, $O_{R} \in O(2 n, \mathbb{R})$ and $K \in \operatorname{Skew}(2 n, \mathbb{R})$ is a real antisymmetric matrix.

The transformations $e^{i K}$ and $O_{R} \in S O(2 n, \mathbb{R})$ can be generated on the $c_{i}$ by applying exponents of bilinears with antisymmetric form using

$$
\text { (a) } \quad c_{m} \Rightarrow e^{-\frac{i}{4} K_{i j} c_{i} c_{j}} c_{m} e^{\frac{i}{4} K_{k l} c_{k} c_{l}}=\left(e^{i K}\right)_{m l} c_{l},
$$

for antisymmetric matrices $K \in \operatorname{Skew}(2 n, \mathbb{C})$. Transformations (a) are not enough, as these are restricted to exponents of antisymmetric matrices, which do not cover all complex orthogonal transformations (A simple example for an orthogonal matrix which cannot be written as an exponent of an antisymmetric matrix is $\sigma_{z} \in O(2, \mathbb{R})$ ).

To get the full $O(2 n, \mathbb{R})$, we need to add similarity transformations that change signature. To generate such transformations we note that conjugation by $c_{k}$ is a similarity transformation since $c_{k}=c_{k}^{-1}$ :

$$
\text { (b) } c_{l} \Rightarrow c_{k} c_{l} c_{k}=-c_{l}+2 \delta_{k l} c_{k} \text {. }
$$

This transformation multiplies all the Majorana operators by -1 except for $c_{k}$ itself. Together (a) and (b) transformations generate all possible $O \in O(2 n, \mathbb{C})$.

Proof of Eq (4). Noting that under the transformation (7), we have $A_{i j} c_{i} c_{j} \rightarrow$ $A_{i j} O_{i k} c_{k} O_{j l} c_{l}=\left(O^{T} A O\right)_{k l} c_{k} c_{l}$, we conclude that for any $O \in O(2 n, \mathbb{C})$ the above similarity transformations preserve the trace, thus we can write:

$$
\mathcal{Z}(A)=\operatorname{Tr} e^{A_{i j} c_{i} c_{j}}=\operatorname{Tr} X e^{A_{i j} c_{i} c_{j}} X^{-1}=\operatorname{Tr} e^{X\left(A_{i j} c_{i} c_{j}\right) X^{-1}}=\mathcal{Z}\left(O A O^{T}\right) .
$$

For A real antisymmetric, one may proceed by transforming A into a canonical form. The eigenvalues of an antisymmetric matrix come in pairs $\pm \epsilon$, and using an (real) orthogonal 
transformation it can be written as:

$$
A=O\left(\oplus_{i} E_{i}\right) O^{-1} \quad ; \quad E_{i}=\left(\begin{array}{cc}
0 & \epsilon_{i} \\
-\epsilon_{i} & 0
\end{array}\right) .
$$

We note that for real symmetric matrices, this type of form is always available: it is essentially the Bogolubov transformation of the modes, and is the one often used when computing the partition function of a paired state. We can now write:

$$
\mathcal{Z}(A)=\operatorname{Tr} e^{\sum \epsilon_{i}\left(c_{2 i} c_{2 i+1}-c_{2 i+1} c_{2 i}\right)}=\prod_{i} 2 \cos 2 \epsilon_{i}
$$

where we used that:

$$
\operatorname{Tr} e^{\epsilon\left(c_{2 i} c_{2 i+1}-c_{2 i+1} c_{2 i}\right)}=2 \cos 2 \epsilon .
$$

Note that:

$$
\operatorname{Pf}\left(e^{E_{i}}-e^{-E_{i}}\right)=\operatorname{Pf}\left(2 \sin \left(\epsilon_{i}\right) i \sigma_{y}\right)=2 \sin \left(\epsilon_{i}\right),
$$

which gives

$$
\left(2 \cos 2 \epsilon_{i}\right)=\frac{\sin \left(4 \epsilon_{i}\right)}{\sin \left(2 \epsilon_{i}\right)}=\frac{\operatorname{Pf}\left(e^{4 E_{i}}-e^{-4 E_{i}}\right)}{\operatorname{Pf}\left(e^{2 E_{i}}-e^{-2 E_{i}}\right)} .
$$

Combining these results together, and using that both Pfaffians and determinants of block matrices are products, we have Eq. (4). Alternatively, we can write

$$
\left(2 \cos 2 \epsilon_{i}\right)^{2}=\left(1+e^{4 i \epsilon_{i}}\right)\left(1+e^{-4 i \epsilon_{i}}\right)=\operatorname{det}\left(1+e^{4 E_{i}}\right)
$$

giving us Eq. (6).

We now show that the form (44) holds also for arbitrary complex antisymmetric matrices $A$, in order to be able to account for complex phases as may appear in mean field superconducting Hamiltonians as well as products of exponents of bilinears.

We claim the formula still holds. Indeed, it follows from the definition of $\mathcal{Z}(A)$, that $\mathcal{Z}(A)$ is manifestly an entire function of the elements $A_{i j}$. On the other hand the Pfaffian expression in (44) is also entire. To see this note that as a ratio of holomorphic functions, the only potentially problematic points are the points where the denominator goes to zero faster than the numerator, making the ratio singular. However, since we have (using $\operatorname{Pf}(M)^{2}=\operatorname{det}(M)$ ) the relation:

$$
\left[\frac{\operatorname{Pf}\left(e^{-4 A}-e^{4 A}\right)}{\operatorname{Pf}\left(e^{-2 A}-e^{2 A}\right)}\right]^{2}=\frac{\operatorname{det}\left(e^{-4 A}-e^{4 A}\right)}{\operatorname{det}\left(e^{-2 A}-e^{2 A}\right)}=\operatorname{det}\left(e^{-2 A}+e^{2 A}\right)
$$


where the right side is manifestly regular, we conclude that this type of singularity can not happen. By analyticity, we conclude that the identity (41) must hold in the complex case as well.

In fact, it is possible to make a stronger argument, in that a canonical form is available even in the case of complex matrices, albeit as a similarity transformation on the Majorana modes, rather than an orthogonal transformation. Indeed, we consider a Gantmacher type decomposition, described in the classic book [41]. This decomposition supplies a complex orthogonal diagonalization of the matrix which is rather complicated: Let $A$ be a rank $r$ anti-symmetric matrix, with elementary divisors $\epsilon_{i}$ and corresponding ranks $f_{i}$, then there exists a matrix $O \in O(2 n, \mathbb{C})$ such that

$$
A=O K O^{-1}
$$

where:

$$
K=\oplus K_{\epsilon_{i}}
$$

the $K$ matrices are of size $2 f_{i}$, and they are of the form

$$
\kappa_{l m}=\frac{1}{2} \begin{cases}-\delta_{l, m+1}=\kappa-\kappa^{T} & m, l<f \\ -i \delta_{m, f-l}-2 \epsilon \delta_{m, f-l+1}-i \delta_{m, f-l+2} & l>f \text { and } m<f \\ \delta_{l, m+1} & m, l>f\end{cases}
$$

For example, for $f=4$ the form is, explicitly:

$$
\frac{1}{2}\left(\begin{array}{cccccccc}
0 & 1 & 0 & 0 & 0 & 0 & i & 2 \epsilon \\
-1 & 0 & 1 & 0 & 0 & i & 2 \epsilon & i \\
0 & -1 & 0 & 1 & i & 2 \epsilon & i & 0 \\
0 & 0 & -1 & 0 & 2 \epsilon & i & 0 & 0 \\
0 & 0 & -i & -2 \epsilon & 0 & -1 & 0 & 0 \\
0 & -i & -2 \epsilon & -i & 1 & 0 & -1 & 0 \\
-i & -2 \epsilon & -i & 0 & 0 & 1 & 0 & -1 \\
-2 \epsilon & -i & 0 & 0 & 0 & 0 & 1 & 0
\end{array}\right)
$$

Since we have shown above that all $O \in O(2 n, \mathbb{C})$ transformations can be lifted as similarity transformations to the Fock space, we can bring any $A$ to this form. Next, instead of 
treating the general Gantmacher form in full generality, we can proceed by first assuming that characteristic numbers are non degenerate. In this case we have $f_{i}=1$ for all $\epsilon_{i}$, the Gantmacher form reduces to the form (12), and one arrives at Eq. (4) directly. We may also remove the condition on non-degeneracy of the eigenvalues of the matrix, by invoking the analyticity argument again. Starting with a degenerate anti-symmetric matrix, we can perturb it with an arbitrarily small deformation into a non-degenerate matrix, where the equality has been established.

Remark 1 . We note that the appearance of square roots is a consequence of the nature of the Majorana representation. The topological reason for this is that the Clifford representation of the Lie algebra of skew-symmetric matrices, even for just one fermion mode (two Majoranas) when exponentiated, corresponds to a double cover. This can be seen in the following simple example: Take $A=0$ and $B_{k l}=2 \pi i\left(\sigma_{y}\right)_{k l}$ if $k, l \in\{1,2\}$ and $B_{k l}=0$ otherwise. Noting that the eigenvalues of $B$ are just $\pm 2 \pi i$, we immediately have:

$$
e^{A}=e^{B}=\mathbf{I}_{2 n},
$$

and

$$
e^{\frac{1}{4} A_{k l} c_{k} c_{l}}=e^{0}=\mathbf{I}_{2^{n}}
$$

where $\mathbf{I}_{2 n}, \mathbf{I}_{2^{n}}$ are the identity matrices in the $2 n$ dimensional Majorana mode space and $2^{n}$ dimensional Fock space, respectively. On the other hand:

$$
e^{\frac{1}{4} B_{k l} c_{k} c_{l}}=e^{\frac{\pi}{2}\left(c_{1} c_{2}-c_{2} c_{1}\right)} \neq \mathbf{I}_{2^{n}}
$$

For example, taking $n=1$, we have:

$$
\operatorname{Tr} e^{\frac{\pi}{2}\left(c_{1} c_{2}-c_{2} c_{1}\right)}=-2=-\operatorname{Tr} 1 .
$$

So when writing $\operatorname{Tr} e^{\frac{1}{4} A_{k l} c_{k} c_{l}}$ in terms of properties of $e^{A}$, the information about the sign comes in a subtle way. This is exactly what our Pfaffian formula (41) keeps track of. Indeed, computing the same expression using the Pfaffian we have

$$
\operatorname{Tr} e^{\frac{\pi}{2}\left(c_{1} c_{2}-c_{2} c_{1}\right)}=\frac{\operatorname{Pf}\left(e^{-2 \pi i \sigma_{y}}-e^{2 \pi i \sigma_{y}}\right)}{\operatorname{Pf}\left(e^{-\pi i \sigma_{y}}-e^{\pi i \sigma_{y}}\right)}=\frac{\operatorname{Pf}(0)}{\operatorname{Pf}(0)} .
$$

To resolve the ratio, we compute the same expression as a limit:

$$
\lim _{\epsilon \rightarrow 0} \operatorname{Tr} e^{\frac{\pi+\epsilon}{2}\left(c_{1} c_{2}-c_{2} c_{1}\right)}=\lim _{\epsilon \rightarrow 0} \frac{\operatorname{Pf}\left(e^{-2(\pi+\epsilon) i \sigma_{y}}-e^{2(\pi+\epsilon) i \sigma_{y}}\right)}{\operatorname{Pf}\left(e^{-(\pi+\epsilon) i \sigma_{y}}-e^{(\pi+\epsilon) i \sigma_{y}}\right)}=\lim _{\epsilon \rightarrow 0} \frac{\operatorname{Pf}\left(-4 \epsilon i \sigma_{y}\right)}{\operatorname{Pf}\left(2 \epsilon i \sigma_{y}\right)}=-2 .
$$




\section{THE TRACE OF A PRODUCT OF EXPONENTS}

For applications it is often more important to understand how to extend the previous results to deal with products of exponents, for example in order to compute expectation values. For this case we derive the following trace formula:

$$
\operatorname{Tr} e^{A_{1 i j} c_{i} c_{j}} \ldots e^{A_{n i j} c_{i} c_{j}}=\sqrt{\operatorname{det}\left(1+e^{4 A_{1}} . . e^{4 A_{n}}\right)} .
$$

Finally, the expectation value of a product of exponents, in a thermal state with a Hamiltonian $H_{i j} c_{k} c_{l}$ is:

$$
\begin{gathered}
\left\langle e^{A_{1 i j} c_{i} c_{j}} \ldots e^{A_{n i j} c_{i} c_{j}}\right\rangle=\sqrt{\operatorname{det}\left(n_{\beta}+\left(1-n_{\beta}\right) e^{4 A_{1}} . . e^{4 A_{n}}\right)}, \\
n_{\beta}=\left(1+e^{4 \beta H}\right)^{-1} .
\end{gathered}
$$

To derive Eq. (27) we first note that:

$$
\left[\frac{1}{4} K_{l m} c_{l} c_{m}, \frac{1}{4} L_{i j} c_{i} c_{j}\right]=\frac{1}{4}([K, L])_{i m} c_{i} c_{m},
$$

which is straightforward to check (see, e.g. [42]). Showing that the map:

$$
K \rightarrow \frac{1}{4} K_{l m} c_{l} c_{m}
$$

is a representation of the Lie algebra of skew-symmetric matrices. In particular it follows that for small enough $|t|<t_{0}$, we can write:

$$
e^{\frac{t}{4} A_{l m} c_{l} c_{m}} e^{\frac{t}{4} B_{l m} c_{l} c_{m}}=e^{\left.\frac{1}{4} C[t A, t B]\right]_{l m} c_{l} c_{m}} \rightarrow e^{t A} e^{t B}=e^{C[t A, t B]},
$$

where $C[t A, t B]$ is given by a Baker Campbell Hausdorff (BCH) type series. We kept $t$ since in the $\mathrm{BCH}$ formula and it's explicit variants such as the Dynkin formula, $C[t A, t B]$ as a series in commutators of $A$ and $B$, has in general a finite radius of convergence (see remark 2 bellow). In this neighborhood, we can immediately write:

$$
\operatorname{Tr} e^{\frac{t}{4} A_{i j} c_{i} c_{j}} e^{\frac{t}{4} B_{i j} c_{i} c_{j}}=\operatorname{Tr} e^{\frac{1}{4} C_{i j} c_{i} c_{j}}=\sqrt{\operatorname{det}\left(1+e^{C}\right)}=\sqrt{\operatorname{det}\left(1+e^{t A} e^{t B}\right)} .
$$

Now we invoke analyticity again: the LHS is an entire function of $t$, and in particular it's square is. On the other hand $\operatorname{det}\left(1+e^{t A} e^{t B}\right)$ is also an entire function, and since the functions are equal for $t<t_{0}$, they are equal everywhere. It is left to take the square root and resolve the sign so as to be an entire function, which goes to $2^{n}$ at $t=0$. Finally, we establish (27) 
by renaming $\{A, B\} \rightarrow\{4 A, 4 B\}$, and repeating the argument iteratively for an arbitrary number of matrices. Unfortunately, the Pfaffian expression Eq. (4), is not available in a simple form anymore, since there is no simple way of expressing the denominator, which requires the square root of $e^{C}$.

Remark 2. The question of the range of analyticity of the $\mathrm{BCH}$ formula has been studied in many works since the classic paper of Wei [43]. The possible non-analyticity of BCH can be demonstrated with an example. Following the approach presented in [43], we construct such an example for our particular Lie algebra of complex skew-symmetric matrices by searching for a pair of such matrices where $[A, B]=A$. The smallest non-commuting algebra of skew-symmetric matrices is of $\operatorname{Skew}(3, \mathbb{C})$. Consider the following pair of anti-symmetric matrices:

$$
A=\left(\begin{array}{ccc}
0 & 1 & 1 \\
-1 & 0 & -i \sqrt{2} \\
-1 & i \sqrt{2} & 0
\end{array}\right) ; B=\left(\begin{array}{ccc}
0 & 1-i \sqrt{2} & 1 \\
-1+i \sqrt{2} & 0 & -1-i \sqrt{2} \\
-1 & 1+i \sqrt{2} & 0
\end{array}\right)
$$

An explicit computation shows that for any scalars $a, b$ :

$$
e^{a(-2 B)} e^{b A}=e^{\left(a(-2 B)+\frac{2 a b e^{2 a}}{e^{2 a}-1} A\right)},
$$

so that

$$
C[-2 a B, b A]=a(-2 B)+\frac{2 a b e^{2 a}}{e^{2 a}-1} A .
$$

Clearly, the BCH $C$ is not analytic in the input matrices $A, B$ close to $a=\pi i$.

To go around this issue, one possible attempt is to use upper triangular matrices $A, B$ instead of antisymmetric ones, since $A_{i j} c_{i} c_{j}=2 A_{i j}^{u p} c_{i} c_{j}$ where $A^{u p}$ is the upper triangular part of $A$.

The algebra of upper triangular matrices is nilpotent, and as such all BCH type series terminate and converge. However, it is straightforward to check that the map $A^{u p} \rightarrow A_{i j}^{u p} c_{i} c_{j}$ is not a representation of the Lie algebra of upper triangular matrices. We do note that upper-triangular matrices $A^{u p}$, can be very useful in obtaining various relations between fermionic traces and determinants or Pfaffians. An example for such is Lieb's theorem on Pfaffians [44]. An alternative derivation of (4) in a manner similar to Lieb's derivation may be possible. 


\section{APPLICATIONS TO FULL COUNTING STATISTICS}

Here, we consider counting statistics in systems evolving with a time dependent mean field BCS type hamiltonian as represented by Bogolubov-de Gennes equations. Consider measuring an observable $Q_{A}$ at time $t=t_{A}$, and measuring an observable $Q_{B}$ at a later time $t=t_{B}$. The usual two-measurement protocol for counting statistics of the difference between the measurement of $Q_{A}$ and $Q_{B}$ is conveniently described by the cumulant generating function:

$$
\chi(\lambda)=\sum_{a, b} p_{a} \operatorname{prob}(a \rightarrow b) e^{-\lambda\left(Q_{A}(a)-Q_{B}(b)\right)},
$$

where $p_{a}=\langle a|\rho| a\rangle$ is the probability to be in state $|a\rangle$ at the initial time $t=t_{A}$, and $\rho$ is the initial density matrix.

From now on, we take $Q_{A}=Q_{B} \equiv Q$, which is the case appearing in most applications (such as charge transport), but the extension to $Q_{A} \neq Q_{B}$ is straightforward. If we take $|a\rangle$ to be a complete set of eigenstates of a (hermitian) operator $\hat{Q}$ with eigenvalues $\hat{Q}|a\rangle=Q(a)|a\rangle$ representing possible outcomes of the observable $Q$, we can write Eq. (37) as

$$
\chi(\lambda)=\Sigma_{a} p_{a}\left\langle a\left|U^{\dagger} e^{\lambda \hat{Q}} U e^{-\lambda \hat{Q}}\right| a\right\rangle,
$$

where $U$ is the evolution operator of the full many body system from $t_{A}$ to $t_{B}$.

In the simplest case, the measured states $|a\rangle$ are also eigenstates of the initial $\rho$. For example, in the case of charge measurements, we take $[\rho, \hat{Q}]=0$. An example for such a situation is a normal lead that is connected at time $t_{A}$ with a superconductor, or where pairing is turned on at time $t_{A}$. In this case Eq. (37) further simplifies to:

$$
\chi(\lambda)=\operatorname{Tr} \rho U^{\dagger} e^{\lambda \hat{Q}} U e^{-\lambda \hat{Q}}=\left\langle U^{\dagger} e^{\lambda \hat{Q}} U e^{-\lambda \hat{Q}}\right\rangle .
$$

To connect with the formalism above, consider fermions in a Fock space built from the single particle Hilbert space $\mathfrak{h}$. We first express bilinear fermion forms in terms of majoranas and then use Eq. (27). Indeed, consider a general bilinear fermion operator as:

$$
\mathcal{M}=\sum_{i j} M_{i j} a_{i}^{\dagger} a_{j}+\frac{1}{2}\left\{\Delta_{-i j} a_{i} a_{j}+\Delta_{+i j} a_{i}^{\dagger} a_{j}^{\dagger}\right\},
$$

where the matrices $\Delta_{-}, \Delta_{+}$are assumed to be anti-symmetric (a choice that can always be made). Note that, in general, we do not demand that the operator $\mathcal{M}$ is hermitian, hence 
we do not assume, a priori, a conjugacy relation between $\Delta_{-}$and $\Delta_{+}$, nor do we assume $M$ to be Hermitian. We rewrite the operator in terms of majoranas as:

$$
\mathcal{M}=\frac{1}{4} \mathbb{M}_{i j} c_{k} c_{l}+\frac{1}{2} \operatorname{Tr} M
$$

with

$$
\mathbb{M}=\left(\begin{array}{cc}
M_{a}+\frac{1}{2}\left(\Delta_{-}+\Delta_{+}\right) & i M_{s}+\frac{i}{2}\left(\Delta_{-}-\Delta_{+}\right) \\
-i M_{s}+\frac{i}{2}\left(\Delta_{-}-\Delta_{+}\right) & M_{a}-\frac{1}{2}\left(\Delta_{-}+\Delta_{+}\right)
\end{array}\right),
$$

where $M_{s / a}=\frac{1}{2}\left(M \pm M^{T}\right)$ are the symmetric and antisymmetric parts of $M$ and $\mathbb{M}$ acts on the space $\mathfrak{h} \otimes \mathbb{C}^{2}$. We then have, by Eq. (27),

$$
\operatorname{Tr} \Pi_{m} e^{\alpha_{m} \mathcal{M}_{m}}=e^{\frac{1}{2} \sum_{m} \operatorname{Tr} M_{m}} \sqrt{\operatorname{det}\left(1+\Pi_{m} e^{\alpha_{m} \mathbb{M}_{m}}\right)} .
$$

When the evolution is governed by a Hamiltonian $\mathcal{H}$, we can write:

$$
\begin{gathered}
\chi(\lambda)=\left\langle e^{+i t \mathcal{H}} e^{\lambda \hat{Q}} e^{-i t \mathcal{H}} e^{-\lambda \hat{Q}}\right\rangle_{\beta}= \\
\operatorname{det}^{1 / 2}\left(n_{\beta}+\left(1-n_{\beta}\right) e^{i t \mathbb{H}} e^{\lambda \mathbb{Q}} e^{-i t \mathbb{H}} e^{-\lambda \mathbb{Q}}\right)
\end{gathered}
$$

with:

$$
n_{\beta}=\frac{1}{1+e^{\beta \mathbb{H}_{0}}}
$$

playing the role of the Fermi function of the initial Hamiltonian $\mathcal{H}_{0}$. For a Hermitian operator $Q$, we see that for real $\lambda$, we must also have $\chi(\lambda)>0$. Therefore, in taking the square root, we take the branch of $\chi(\lambda)$ which is real and positive everywhere on the real $\lambda$ axis.

Remark 3. In the case where the initial state is not diagonal in eigenstates of the charge operator considered, one has to replace the initial density matrix $\rho$ by a "decohered" initial density matrix in the charge basis $\tilde{\rho}$ :

$$
\rho \rightarrow \tilde{\rho}=\sum_{a}\langle a|\rho| a\rangle|a\rangle\langle a|
$$

which can be, for example, implemented by an auxiliary integral. 


\section{FULL COUNTING STATISTICS OF THE NUMBER OF FERMIONS IN A REGION}

For certain applications one does not need two measurements, for example when considering measuring the full counting statistics of a given observable. In such a case we can extract the probability distribution from an expression of the form:

$$
\chi(\lambda)=\sum_{a} p_{a} e^{\lambda Q_{A}(a)}=\left\langle e^{\lambda Q}\right\rangle
$$

As an example for such a situation, let us consider the counting statistics of the number of particles in a region $A$ of a thermal state defined by a BCS type Hamiltonian:

$$
\mathcal{H}=\sum_{i j} H_{i j} a_{i}^{\dagger} a_{j}+\frac{1}{2} \Delta_{i j} a_{i} a_{j}+\text { h.c. }
$$

Using the hermiticity of $\mathcal{H}$ and (41) we associate with $\mathcal{H}$ the matrix $\mathbb{H}_{0}$ given by:

$$
\mathbb{H}_{0}=\left(\begin{array}{cc}
i \operatorname{Im}(H+\Delta) & i \operatorname{Re}(\Delta+H) \\
i \operatorname{Re}(\Delta-H) & i \operatorname{Im}(H-\Delta)
\end{array}\right)
$$

Noting that the number operator in region $A$ can be written, using Eq. (41), as

$$
\hat{N}_{A}=\sum_{i \in A} a_{i}^{\dagger} a_{i}=\sum_{i, j} P_{A i, j} a_{i}^{\dagger} a_{j}=-\sum_{i, j} \frac{1}{4}\left(P_{A} \otimes \sigma_{y}\right)_{i j} c_{i} c_{j}+\frac{1}{2} \operatorname{Tr} P_{A}
$$

where $P_{A i, j}=\delta_{i j} \Theta(i \in A)$ are the matrix elements of $P_{A}$, the single particle projector on region $A$. We have

$$
\begin{gathered}
\chi_{A}(\lambda) \equiv \frac{\operatorname{Tr} e^{-\beta \mathcal{H}_{0} e^{\lambda \hat{N}_{A}}}}{\mathcal{Z}}= \\
e^{\frac{1}{2} \lambda \operatorname{Tr} P_{A}} \sqrt{\operatorname{det}\left(\frac{1}{1+e^{\beta \mathbb{H}_{0}}}+\left(1-\frac{1}{1+e^{\beta \mathbb{H}_{0}}}\right) e^{-\lambda P_{A} \otimes \sigma_{y}}\right)}
\end{gathered}
$$

where $\sigma_{y}$ acts on the auxiliary $\mathbb{C}^{2}$ space, so

$$
P_{A} \otimes \sigma_{y}=\left(\begin{array}{cc}
0 & i P_{A} \\
-i P_{A} & 0
\end{array}\right)
$$

We note that here the sign of the square root of the determinant is unambiguous: as before, the sign of $\chi_{A}(\lambda)$ is assured to be positive for real $\lambda$ by it's definition, Eq. (50).

As a an example, for simplicity, we consider a BCS type Hamiltonian (48), and we simply take $A$ to be the entire volume of the system, allowing us to work in momentum space. 
Written in a momentum state basis we will take $H=\sum_{p} h(p) a_{p}^{\dagger} a_{p}$, where $h(p)$ may, for example, be taken the form $h(p)=\frac{p^{2}}{2 m}-\mu$. Also, we take a simple $s$-wave superconducting gap $\Delta$, only pairing states with momenta $p$ and $-p$. In this case we have to consider each pair of $p,-p$ separately. For each paired couple with a given $p$, we must consider a $4 \times 4$ block from $\mathbb{H}_{0}$ of the form:

$$
\left(\begin{array}{cccc}
0 & 0 & h(p) & -\Delta(p) \\
0 & 0 & \Delta(p) & h(p) \\
-h(p) & -\Delta(p) & 0 & 0 \\
\Delta(p) & -h(p) & 0 & 0
\end{array}\right)
$$

Plugging the matrix (51) into (50), and doing some algebra we find:

$$
\begin{gathered}
\chi(\lambda, p)=\frac{e^{\lambda}}{2} \operatorname{sech}^{2}\left(\frac{\beta}{2} \sqrt{h^{2}+\Delta^{2}}\right) \times \\
\left(1+\cosh \left(\beta \sqrt{h^{2}+\Delta^{2}}\right) \cosh (\lambda)-\frac{h \sinh \left(\beta \sqrt{h^{2}+\Delta^{2}}\right) \sinh (\lambda)}{\sqrt{h^{2}+\Delta^{2}}}\right),
\end{gathered}
$$

and finally:

$$
\begin{gathered}
\log (\chi(\lambda))=\int g(p) d p\{\lambda+\log (1+ \\
\left.\cosh \left(\beta \sqrt{h^{2}+\Delta^{2}}\right) \cosh (\lambda)-\frac{h \sinh \left(\beta \sqrt{h^{2}+\Delta^{2}}\right) \sinh (\lambda)}{\sqrt{h^{2}+\Delta^{2}}}\right)- \\
\left.\log \left(2 \cosh ^{2}\left(\frac{1}{2} \beta \sqrt{h^{2}+\Delta^{2}}\right)\right)\right\},
\end{gathered}
$$

where $g(p)$ is the density of pairs at a given $p$.

To verify our result, we can also derive this formula directly. Indeed, write the pairing Hamiltonian (suppressing the momentum index $p$ ),

$$
\mathcal{H}=h\left(a^{\dagger} a+b^{\dagger} b\right)+\Delta\left(a^{\dagger} b^{\dagger}+b a\right) .
$$

Affecting a Bogolubov transformation we can write $\mathcal{H}$ in the form

$$
\mathcal{H}=\mathcal{E} c^{\dagger} c-\mathcal{E} d^{\dagger} d \quad ; \quad \mathcal{E}=\sqrt{h^{2}+\Delta^{2}}
$$

with

$$
c=u a+v b^{\dagger} \quad ; \quad d=v a-u b^{\dagger},
$$

where

$$
u=\cos \left(\frac{1}{2} \tan ^{-1}\left(\frac{\Delta}{h}\right)\right) ; v=\sin \left(\frac{1}{2} \tan ^{-1}\left(\frac{\Delta}{h}\right)\right) .
$$


Taking the number operator of the pair $\hat{N}_{A}=a^{\dagger} a+b^{\dagger} b$, we have

$$
\begin{gathered}
\left\langle e^{\lambda \hat{N}}\right\rangle=\left\langle\left(1+(z-1) a^{\dagger} a\right)\left(1+(z-1) b^{\dagger} b\right)\right\rangle= \\
\left(1-\left\langle a^{\dagger} a\right\rangle-\left\langle b^{\dagger} b\right\rangle+\left\langle a^{\dagger} a b^{\dagger} b\right\rangle\right)+ \\
z\left(\left\langle a^{\dagger} a\right\rangle+\left\langle b^{\dagger} b\right\rangle-2\left\langle a^{\dagger} a b^{\dagger} b\right\rangle\right)+z^{2}\left\langle a^{\dagger} a b^{\dagger} b\right\rangle,
\end{gathered}
$$

where we denoted $z=e^{\lambda}$. Substituting $a=u c-v d$ and $b^{\dagger}=v c+u d$ and computing the resulting thermal correlations, which are just free fermions in terms of the $c$ and $d$ operators, we find:

$$
\begin{gathered}
\chi=\left(v^{2} n_{c}+u^{2} n_{d}-n_{c} n_{d}\right)+z\left(1-n_{d}-n_{c}+2 n_{d} n_{c}\right)+ \\
z^{2}\left(v^{2} n_{d}+u^{2} n_{c}-n_{d} n_{c}\right)
\end{gathered}
$$

with $n_{d}, n_{c}$ the fermi functions for $c, d$, i.e.:

$$
n_{d}=\frac{1}{1+e^{-\beta \mathcal{E}}} \quad ; \quad n_{c}=\frac{1}{1+e^{\beta \mathcal{E}}}
$$

After substituting $z$ and $\mathcal{E}$, and some tedious algebra we recover the result (52).

\section{OVERLAPS OF PAIRED STATES AND COUNTING STATISTICS OF CHARGE}

Here we show how to compute the overlap between different BCS like states built out of the vacuum. Such a state is built out of application of pair creation to the vacuum. We will write a (un-normalized) state of this type in the form:

$$
|D\rangle=e^{D_{i j} a_{i}^{\dagger} a_{j}^{\dagger}}|0\rangle
$$

where the state $|0\rangle$ is the vacuum state, so that $a_{i}|0\rangle=0$ for all $i$, and $D$ is an $n \times n$ antisymmetric matrix. We now derive the following formulas:

$$
\left\langle D^{\prime} \mid D\right\rangle=(-1)^{n} \operatorname{Pf}\left(\begin{array}{cc}
D^{\prime \dagger} & -I_{n} \\
I_{n} & D
\end{array}\right)
$$

and as a corollary:

$$
\left\langle e^{i \lambda N_{A}}\right\rangle_{D}=\frac{(-1)^{n}}{\operatorname{det}\left(1+D^{\dagger} D\right)} \operatorname{Pf}\left(\begin{array}{cc}
D^{\dagger} & -I_{n} \\
I_{n} & e^{i \lambda P_{A}} D e^{i \lambda P_{A}}
\end{array}\right) .
$$


To derive these relations, we first note that $|0\rangle$ is the ground state of the Hamiltonian $H=\hat{N}=\sum_{i} a_{i}^{\dagger} a_{i}$. We can therefore write the overlap in the following way:

$$
\left\langle D^{\prime} \mid D\right\rangle=\lim _{\beta \rightarrow \infty} \frac{1}{Z} \operatorname{Tr}\left[e^{D_{i j} a_{i}^{\dagger} a_{j}^{\dagger}} e^{-\beta \hat{N}}\left(e^{D_{i j}^{\prime} a_{i}^{\dagger} a_{j}^{\dagger}}\right)^{\dagger}\right] .
$$

Going to the Majorana representation we have:

$$
\begin{gathered}
D_{i j} a_{i}^{\dagger} a_{j}^{\dagger}=\frac{1}{8}\left[D \otimes\left(\sigma_{z}-i \sigma_{x}\right)\right]_{\alpha \beta} c_{\alpha} c_{\beta}, \\
D_{i j} a_{i} a_{j}=\frac{1}{8}\left[D \otimes\left(\sigma_{z}+i \sigma_{x}\right)\right]_{\alpha \beta} c_{\alpha} c_{\beta}, \\
\Sigma_{i} a_{i}^{\dagger} a_{i}=-\frac{1}{4} I_{n} \otimes \sigma_{y}+\frac{1}{2} \operatorname{Tr} I_{n} .
\end{gathered}
$$

We have seen above (27), that:

$$
\frac{1}{Z} \operatorname{Tr} e^{-\beta N}\left(e^{D_{i j}^{\prime} a_{i}^{\dagger} a_{j}^{\dagger}}\right){ }^{\dagger} e^{D_{i j} a_{i}^{\dagger} a_{j}^{\dagger}}=\sqrt{\operatorname{det}\left(1-n_{\beta}+n_{\beta} e^{\frac{1}{2}\left(D^{\prime \dagger} \otimes\left(\sigma_{z}+\mathrm{i} \sigma_{x}\right)\right)} e^{\frac{1}{2}\left(D \otimes\left(\sigma_{z}-\mathrm{i} \sigma_{x}\right)\right)}\right)} .
$$

To proceed we make several observations.

1. Notice that $\left(\sigma_{z} \pm \mathrm{i} \sigma_{x}\right)^{2}=0$ are nilpotent, allowing us to write:

$$
e^{\frac{1}{2}\left(D^{\prime \dagger} \otimes\left(\sigma_{z}+\mathrm{i} \sigma_{x}\right)\right)} e^{\frac{1}{2}\left(D \otimes\left(\sigma_{z}-\mathrm{i} \sigma_{x}\right)\right)}=\left(1+\frac{1}{2}\left(D^{\prime \dagger} \otimes\left(\sigma_{z}+\mathrm{i} \sigma_{x}\right)\right)\right)\left(1+\frac{1}{2}\left(D \otimes\left(\sigma_{z}-\mathrm{i} \sigma_{x}\right)\right)\right)
$$

2. Using the limit:

$$
\lim _{\beta \rightarrow \infty} n_{\beta}=\lim _{\beta \rightarrow \infty} \frac{1}{1+e^{4 \beta\left(-\frac{1}{4} I_{n} \otimes \sigma_{y}\right)}}=\frac{1}{2}\left(I_{2 n}+I_{n} \otimes \sigma_{y}\right)=\frac{1}{2} I_{n} \otimes\left(1+\sigma_{y}\right) \equiv P_{+},
$$

we have

$$
\left\langle D \mid D^{\prime}\right\rangle=\sqrt{\operatorname{det}\left(P_{+}+P_{+}\left(1+\frac{1}{2}\left(D^{\prime \dagger} \otimes\left(\sigma_{z}+\mathrm{i} \sigma_{x}\right)\right)\right)\left(1+\frac{1}{2}\left(D \otimes\left(\sigma_{z}-\mathrm{i} \sigma_{x}\right)\right)\right) P_{+}\right)}
$$

3. Finally, we use:

$$
P_{+}\left(\sigma_{z} \pm \mathrm{i} \sigma_{x}\right) P_{+}=0
$$

and

$$
P_{+}\left(\sigma_{z}+\mathrm{i} \sigma_{x}\right)\left(\sigma_{z}-\mathrm{i} \sigma_{x}\right) P_{+}=2 I_{n} \otimes\left(1+\sigma_{y}\right)=4 P_{+}
$$

Combining the above observations we find:

$$
\left\langle D \mid D^{\prime}\right\rangle=\sqrt{\operatorname{det}\left(P_{+}\left(1+D^{\prime \dagger} D \otimes I_{2}\right) P_{+}\right)}=\sqrt{\operatorname{det}_{n}\left(1+D^{\prime \dagger} D\right)} .
$$


Using the rules of determinants of block matrices we can also rewrite the last expression as:

$$
\left\langle D \mid D^{\prime}\right\rangle=\sqrt{\operatorname{det}\left(\begin{array}{cc}
D^{\prime \dagger} & -I \\
I & D
\end{array}\right)} .
$$

We now finally have a determinant of an anti-symmetric matrix, and the identity $P f(A)^{2}=$ $\operatorname{det}(A)$ (valid for anti-symmetric matrices, but not true for general matrices) can be safely used, to get:

$$
\left\langle D \mid D^{\prime}\right\rangle=(-1)^{n} \operatorname{Pf}\left(\begin{array}{cc}
D^{\prime \dagger} & -I \\
I & D
\end{array}\right)
$$

where the phase $(-1)^{n}$ was added by demanding $\langle 0 \mid 0\rangle=1$ and using:

$$
\operatorname{Pf}\left(\begin{array}{cc}
0 & -I_{n} \\
I_{n} & 0
\end{array}\right)=(-1)^{n}
$$

which establishes Eq. (62).

Finally, to get equation (

$$
\left\langle e^{i \lambda N_{A}}\right\rangle_{D}=\frac{1}{\langle D \mid D\rangle}\left\langle D\left|e^{i \lambda N_{A}}\right| D\right\rangle=\frac{1}{\langle D \mid D\rangle}\left\langle D \mid e^{i \lambda P_{A}} D e^{i \lambda P_{A}}\right\rangle
$$

where the last equation is a consequence of

$$
e^{i \lambda N_{A}} a_{i}^{\dagger} e^{-i \lambda N_{A}}=\left\{\begin{array}{cc}
e^{i \lambda} a_{i}^{\dagger} & i \in A \\
a_{i}^{\dagger} & i \notin A
\end{array} .\right.
$$

Now using (62) in (78) gives (63).

\section{PARITY FLUCTUATIONS}

Consider the distribution of the parity operator applied to a subset $A$ of modes of the (complex-fermions) $a_{i}$. In systems with pairing, such parity measurements may be associated with topological effects, and are affected by the presence of majorana zero modes, see, e.g. [45]. The parity operator can be written as:

$$
\mathcal{P}=(-1)^{\hat{N}_{A}}=e^{\pi i \hat{N}_{A}} .
$$


Noting that $\langle\mathcal{P}\rangle=p_{\mathrm{e}}-p_{\mathrm{o}}=2 p_{\mathrm{e}}-1$, where $p_{\mathrm{e}}, p_{\mathrm{o}}$ is the probability that the parity of the fermion number is even/odd. It's variance is given by:

$$
\sigma^{2}=\left\langle\mathcal{P}^{2}\right\rangle-\langle\mathcal{P}\rangle^{2}=1-\langle\mathcal{P}\rangle^{2}
$$

We immediately see that:

$$
\sigma^{2}=1-\langle\mathcal{P}\rangle^{2}=1-\operatorname{det}\left(1-n_{\beta}+n_{\beta} e^{-i \pi \sigma_{y} P_{A}}\right)
$$

This expression can be further simplified using:

$$
e^{-i \pi \sigma_{y} P_{A}}=\mathbf{I}_{2 n}-2 P_{A} \otimes \mathbf{I}_{2}
$$

to get

$$
\left.\sigma^{2}=1-\operatorname{det}\left(1-2 n_{\beta} P_{A} \otimes \mathbf{I}_{2}\right)\right)
$$

The expectation value of the parity itself can be computed for states such as $|D\rangle$ in (63) by simply plugging in $\lambda=\pi$. One can also derive Pfaffian forms for the case not covered in (63), however here we will proceed in the simplest way using the determinant formulas above.

We can write it down in the following way. If $\hat{N}_{A}=\sum_{m \in A} \hat{N}_{m}$ for some set of modes, with $\hat{N}_{m}$ is the number operator associated with mode $m$, then we use the relation

$$
e^{i \pi \hat{N}_{m}}=1-2 \hat{N}_{m}=\left.\left(1-2 \partial_{\lambda}\right) e^{\lambda \hat{N}_{m}}\right|_{\lambda=0},
$$

to write

$$
\langle\mathcal{P}\rangle=\frac{1}{Z} \operatorname{Tr} e^{-\beta H_{i j} c_{k} c_{l}} \prod_{m \in A} e^{i \pi \hat{N}_{m}}=\left.\frac{1}{Z} \prod_{m \in A}\left(1-2 \frac{\partial}{\partial \lambda_{i}}\right) \operatorname{Tr} e^{-\beta H_{i j} c_{k} c_{l}} \prod_{k \in A} e^{\lambda_{k} \hat{N}_{k}}\right|_{\{\lambda\}=0} .
$$

We can finally write this expression as:

$$
\langle\mathcal{P}\rangle=\left.\prod_{m \in A}\left(1-2 \frac{\partial}{\partial \lambda_{m}}\right) e^{\frac{1}{2} \sum_{i \in A} \lambda_{i}} \operatorname{det}^{1 / 2}\left(n_{\beta}+\left(1-n_{\beta}\right) e^{\sum_{i \in A} \lambda_{i} \sigma_{y} P_{i}}\right)\right|_{\{\lambda\}=0} .
$$

Note that there is no sign ambiguity in this last expression since for Hermitian $H$ and real $\lambda$, $\operatorname{Tr} e^{-\beta H_{i j} c_{k} c_{l}} \prod_{m \in A} e^{\lambda_{i} \hat{N}_{m}}$ should always be positive. In topological applications one considers Majorana zero modes as the simplest known example of non-abelian particles, $P_{A}$ can be taken to be the rank one projection operator on the Dirac fermion mode consisting of the two unpaired Majoranas, and thus their "state" is determined by the parity. For this type of applications the formula above works very well, since we deal with a particular mode or two, and one can analytically carry out the derivatives above. 


\section{FINAL REMARKS}

In this paper we have derived formulas for the traces of exponentials of fermion bilinears which include pairing terms, and are not necessarily hermitian. We are not aware of previous appearance in the literature of the "sign ambiguity free" Pfaffian formulas Eq. (44) and Eq. (63), nor of a general presentation of Eq. (27) and Eq. (28), rather than treatment of special cases.

We believe that the (perhaps more practically useful) expressions such as Eq. (27) and Eq. (28) may have appeared in various forms in dealing with concrete problems, however we feel it is useful to give them a general framework and a simple proof, and make them available for other types of problems. Indeed, the above expressions can be straightforwardly applied to numerical and analytical investigations of time dependent problems involving fermions, such as the extension of the study of quasi-particle modeling of X-ray absorption in the cuprates [46, 47] to take into account the presence of pairing terms [48].

\section{Acknowledgement}

I am grateful to Eugene Demler and David Benjamin for discussions, and to Gian Michele Graf for reference [44]. The work was supported by the NSF CAREER grant DMR-0956053.

[1] L. Levitov and G. Lesovik, JETP Letters 55, 555 (1992).

[2] C. Beenakker and H. Schomerus, Physical Review Letters 86, 700 (2001).

[3] Y. M. Blanter and M. Buttiker, Physics Reports 336, 1 (2000).

[4] Y. Nazarov, Quantum noise in mesoscopic physics (Springer, 2003).

[5] Y. Nazarov and M. Kindermann, The European Physical Journal B-Condensed Matter and Complex Systems 35, 413 (2003).

[6] K. Schönhammer, Physical Review B 75, 205329 (2007).

[7] M. Esposito, U. Harbola, and S. Mukamel, Rev. Mod. Phys. 81, 1665 (2009).

[8] A. Polkovnikov, E. Altman, and E. Demler, Proc. Natl. Acad. Sci. 103, 6125 (2006).

[9] V. Gritsev, E. Altman, E. Demler, and A. Polkovnikov, Nature Physics 2, 705 (2006).

[10] I. Klich, Invited contribution to "Quantum Noise in Mesoscopic Physics", edited by Yu. V. Nazarov and Ya. M. Blanter (2003). 
[11] B. Muzykantskii, N. d'Ambrumenil, and B. Braunecker, Physical Review Letters 91, 266602 (2003).

[12] D. Abanin and L. Levitov, Physical Review Letters 94, 186803 (2005).

[13] M. Kulkarni, K. L. Tiwari, and D. Segal, New Journal of Physics 15, 013014 (2013).

[14] D. Ivanov and A. Abanov, arXiv preprint arXiv:1203.6325 (2012).

[15] S. Genway, J. M. Hickey, J. P. Garrahan, and A. D. Armour, arXiv preprint arXiv:1212.5200 (2012).

[16] V. Eisler and Z. Rácz, Physical Review Letters 110, 060602 (2013).

[17] L. Levitov and M. Reznikov, Physical Review B 70, 115305 (2004).

[18] G. Gershon, Y. Bomze, E. Sukhorukov, and M. Reznikov, Physical Review Letters 101, 16803 (2008).

[19] Y. Bomze, G. Gershon, D. Shovkun, L. Levitov, and M. Reznikov, Physical Review Letters 95, 176601 (2005).

[20] T. Fujisawa, T. Hayashi, R. Tomita, and Y. Hirayama, Science 312, 1634 (2006).

[21] B. Muzykantskii and D. Khmelnitskii, Physical Review B 50, 3982 (1994).

[22] W. Belzig and Y. V. Nazarov, Physical Review Letters 87, 197006 (2001).

[23] P. Samuelsson, G. Johansson, Å. Ingerman, V. Shumeiko, and G. Wendin, Physical Review B 65, $180514(2002)$.

[24] A. Braggio, M. Governale, M. G. Pala, and J. König, Solid State Communications 151, 155 (2011).

[25] V. F. Maisi, D. Kambly, C. Flindt, and J. P. Pekola, Physical Review Letters 112, 036801 (2014).

[26] C. Kane and M. P. Fisher, Physical Review Letters 72, 724 (1994).

[27] E. Berg, Y. Oreg, E.-A. Kim, and F. von Oppen, Physical Review Letters 102, 236402 (2009).

[28] M. Milletarì and B. Rosenow, Physical Review Letters 111, 136807 (2013).

[29] R. De-Picciotto, M. Reznikov, M. Heiblum, V. Umansky, G. Bunin, and D. Mahalu, Nature 389, 162 (1997).

[30] L. Saminadayar, D. Glattli, Y. Jin, and B. c.-m. Etienne, Physical Review Letters 79, 2526 (1997).

[31] H. Inoue, A. Grivnin, N. Ofek, M. Heiblum, V. Umansky, and D. Mahalu, arXiv preprint arXiv:1310.0691 (2013). 
[32] W. De Roeck, Comptes Rendus Physique 8, 674 (2007).

[33] J. Avron, S. Bachmann, G. Graf, and I. Klich, Communications in Mathematical Physics 280, 807 (2008).

[34] J. Dereziński, W. De Roeck, and C. Maes, Journal of Statistical Physics 131, 341 (2008).

[35] V. Jaksic, Y. Ogata, Y. Pautrat, C.-A. Pillet, et al., Quantum Theory from Small to Large Scales pp. 213-410 (2012).

[36] D. Bernard and B. Doyon, Journal of Mathematical Physics 53, 122302 (2012).

[37] I. Klich, G. Refael, and A. Silva, Physical Review A 74, 32306 (2006).

[38] I. Klich and L. Levitov, Physical Review Letters 102, 100502 (2009).

[39] H. Song, C. Flindt, S. Rachel, I. Klich, and K. Le Hur, Physical Review B 83, 161408 (2011).

[40] H. F. Song, S. Rachel, C. Flindt, I. Klich, N. Laflorencie, and K. Le Hur, Physical Review B 85, 035409 (2012).

[41] F. Gantmacher, Applications of the Theory of Matrices (Interscience, New York, 1959).

[42] A. Kitaev, Annals of Physics 321, 2 (2006).

[43] J. Wei, Journal of Mathematical Physics 4, 1337 (1963).

[44] E. H. Lieb, Journal of Combinatorial Theory 5, 313 (1968).

[45] F. Burnell, A. Shnirman, and Y. Oreg, Physical Review B 88, 224507 (2013).

[46] D. Benjamin, D. Abanin, P. Abbamonte, and E. Demler, Physical Review Letters 110, 137002 (2013).

[47] D. Benjamin, I. Klich, and E. Demler, Physical Review Letters 112, 247002 (2014).

[48] D. Benjamin, I. Klich, and E. Demler, In preparation (2014). 